Pacific Journal of Mathematic 


\title{
APPROXIMATION OF COMPACT HOMOGENEOUS MAPS
}

\author{
JoHN R. HubBaRD
}

\begin{abstract}
Within the class of continuous homogeneous maps between Banach spaces, it is proved that every compact map can be uniformly approximated by finite-rank maps. This result is obtained by means of the classical metric projection on Banach spaces.
\end{abstract}

The classical approximation problem is to determine those Banach spaces on which every compact continuous linear map can be uniformly approximated by finite-rank continuous linear maps. P. Enflo [1] recently constructed a Banach space on which this approximation is not possible.

Instead of restricting the class of underlying spaces in order to obtain the approximation property, one can expand the class of continuous maps by weakening the linearity requirement. Let $E$ and $F$ be Banach spaces over some field, and let $\mathscr{H}(E, F)$ denote the Banach space of all continuous homogeneous maps from $E$ into $F$. The norm on $\mathscr{H}(E, F)$ is the uniform norm: $\|T\|=\sup \{\|T x\|:\|x\| \leqq 1\}$.

THEOREM. If $T \in \mathscr{H}(E, F)$ is compact, then for each $\epsilon>0$ there exists a finite-rank map $P \in \mathscr{H}(E, F)$ such that $\|T-P\|<\epsilon$.

The proof is developed through several elementary lemmas. The first two of these are well-known and can be found in Köthe [3].

LeMma 1. If $(G,\|\cdot\|)$ is a separable Banach space, then there exists a strictly convex norm $\|\cdot\|_{1}$ and a number $\rho>0$ such that $\|w\|<\rho\|w\|_{1}$, for all $w \in G$.

LEMMA 2. If $G$ is a strictly convex Banach space and if $M$ is a finite-dimensional subspace, then there exists a map $P_{M} \in \mathscr{H}(G, M)$ such that

$$
\left\|y-P_{M} y\right\|=\min \{\|y-z\|: z \in M\}
$$

for all $y \in G$.

The map $P_{M}: G \rightarrow M$ is usually called the metric projection of $G$ onto $M$. Köthe [3] calls it the "nearest-point mapping". 
LEMMA 3. If $B$ is a relatively compact subset of $G$, then for each $\delta>0$ there exists a finite-dimensional subspace $M$ of $G$ such that

$$
\sup \{\min \{\|y-z\|: z \in M\}: y \in B\} \leqq \delta .
$$

Proof. Let $\left\{y_{1}, \cdots, y_{k}\right\} \subset B$ such that $y \in B \Rightarrow\left\|y-y_{j}\right\|<\delta$ for some $j \leqq k$. Let $M=\operatorname{sp}\left\{y_{1}, \cdots, y_{k}\right\}$ so that $\operatorname{dim} M \leqq k$. Then for each $y \in B, \min \{\|y-z\|: z \in M\}<\delta$; thus,

$$
\sup \{\min \{\|y-z\|: z \in M\}: y \in B\} \leqq \delta .
$$

LEMMA 4. If $C$ is a balanced convex subset of $G$, then its linear span $\operatorname{sp} C=\bigcup_{n=1}^{\infty} n C$.

Proof. Obviously sp $C \supset \bigcup_{n=1}^{\infty} n C$. If $w \in \operatorname{sp} C$, then since $C$ is balanced, $w=\sum_{j=1}^{k} \beta, w_{l}$, where all $\beta, \neq 0, w_{l} \in C$ and $\left\|w_{j}\right\| \leqq 1$. Let $y=\beta^{-1} w$ where $\beta=\sum_{j=1}^{k}\left|\beta_{j}\right|$; then $y=\beta^{-1} \sum_{j=1}^{k} \beta_{j} w_{j}=\sum_{j=1}^{k} \alpha_{j} v_{j}$ where $\alpha_{\jmath}=\beta^{-1}\left|\beta_{j}\right|$ and $v_{\jmath}=\left|\beta_{j}\right|^{-1} \beta_{j} w_{j}$. Now each $v_{j} \in C$, since $C$ is balanced, and $\sum_{j=1}^{k} \alpha_{j}=1$. Thus $y \in C$, since $C$ is convex. Thus $w=$ $\beta y \in \cup_{n=1}^{\infty} n C$.

LEMmA 5. If $T \in \mathscr{H}(E, F)$ is compact, then there exists a closed, separable subspace $G$ of $F$ such that $T \in \mathscr{H}(E, G)$.

Proof. Let $C$ be the convex hull of $T(U)$, the image of the closed unit ball $U$ of $E$. Let $G=\operatorname{cl}\left(\bigcup_{n=1}^{\infty} n C\right)$, the closure of $\bigcup_{n=1}^{\infty} n C$ in $F$. Now $C$ is balanced, since $T$ is homogeneous. Thus by Lemma 4, $G=\mathrm{cl}(\operatorname{sp} C)$. Now $T(U)$ is relatively compact, so $G$ is separable. Finally, $T(E) \subset G$ because $x \in E \Rightarrow T x=T(\|x\| u)=$ $\|x\| T u$, where $x=\|x\| u$ for some $u \in U, \Rightarrow T x \in\|x\| C \subset \bigcup_{n=1}^{\infty} n C \subset G$.

Proof of Theorem. By Lemma 5, $T \in \mathscr{H}(E, G)$, where $G$ is separable. Then by Lemma $1, G$ has a strictly convex norm $\|\cdot\|_{1}$ and a number $\rho>0$ such that $\|w\|<\rho\|w\|_{1}, \forall w \in G$. Let $\epsilon>0$ and set $\delta=\rho^{-1} \epsilon>0$. Let $B=T(U)$. Then by Lemma 3, there exists a finitedimensional subspace $M$ of $G$ such that

$$
\sup \left\{\min \left\{\|y-z\|_{1}: z \in M\right\}: y \in B\right\} \leqq \delta .
$$

Then by Lemma 2 , there exists a map $P_{M} \in \mathscr{H}(G, M)$ such that

$$
\left\|y-P_{M} y\right\|_{1}=\min \left\{\|y-z\|_{1}: z \in M\right\}
$$

for each $y \in G$. Let $P=P_{M} \circ T$. Then $P \in \mathscr{H}(E, M)$, and 


$$
\begin{aligned}
x \in U & \Rightarrow\|T x-P x\|<\rho\|T x-P x\|_{1} \\
& =\rho\left\|T x-P_{M}(T x)\right\|_{1}=\rho \min \left\{\|T x-z\|_{1}: z \in M\right\} \\
& \leqq \rho \sup \left\{\min \left\{\|y-z\|_{1}: z \in M\right\}: y \in B\right\} \leqq \rho \delta=\epsilon .
\end{aligned}
$$

COROLlary. Every compact continuous linear map can be uni. formly approximated by finite-rank continuous homogeneous maps.

\section{REFERENCES}

1. P. Enflo, A counterexample to the approximation property in Banach spaces, Acta Math., 130, (1973), 309-317.

2. J. R. Hubbard, Homogeneous maps between Banach spaces, Ph.D. thesis, The University of Michigan, 1973.

3. G. Köthe, Topological Vector Spaces I, Springer-Verlag, New York, 1969.

Received September 15, 1974. This research constituted part of the author's doctoral thesis [2], written at The University of Michigan under the direction of Professor M. S. Ramanujan. The author wishes to thank Professor Ramanujan for his friendly guidance and encouragement during the preparation of the thesis.

LyCOMING COLLEGE 




\section{Pacific Journal of Mathematics}

\section{Vol. 62, No. $1 \quad$ January, 1976}

Mieczyslaw Altman, Contractor directions, directional contractors and

directional contractions for solving equations . .................. 1

Michael Peter Anderson, Subgroups of finite index in profinite groups .........

Zvi Arad, Abelian and nilpotent subgroups of maximal order of groups of odd order

John David Baildon and Ruth Silverman, On starshaped sets and Helly-type theorems ..........................................

John W. Baker and R. C. Lacher, Some mappings which do not admit an

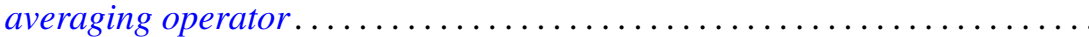

Joseph Barback, Composite numbers and prime regressive isols . . . . . . . . . .

David M. Boyd, Composition operators on $H^{p}(A) \ldots \ldots \ldots \ldots \ldots \ldots \ldots$

Maurice Chacron, Co-radical extension of PI rings . . . . . . . . . . . . .

Fred D. Crary, Some new engulfing theorems . . . . . . . . . . . . . . .

Victor Dannon and Dany Leviatan, A representation theorem for convolution transform with determining function in $L^{p} \ldots \ldots \ldots \ldots \ldots \ldots \ldots \ldots \ldots \ldots \ldots \ldots \ldots \ldots$

Mahlon M. Day, Lumpy subsets in left-amenable locally compact

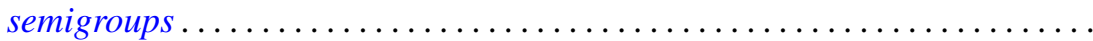

Michael A. Gauger, Some remarks on the center of the universal enveloping algebra of a classical simple Lie algebra . .

David K. Haley, Equational compactness and compact topologies in rings

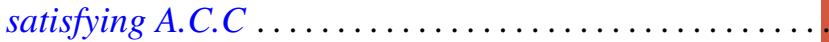

Raymond Heitmann, Generating ideals in Prüfer domains .

Gerald Norman Hile, Entire solutions of linear elliptic equations with

Laplacian principal part. .

Richard Oscar Hill, Moore-Postnikov towers for fibrations in which $\pi_{1}$ (fiber) is non-abelian

John Rast Hubbard, Approximation of compact homogeneous maps . .

Russell L. Merris, Relations among generalized matrix functions . .

V. S. Ramamurthi and Edgar Andrews Rutter, On cotorsion radicals ...

Ralph Tyrrell Rockafellar and Roger Jean-Baptiste Robert Wets, Stochastic

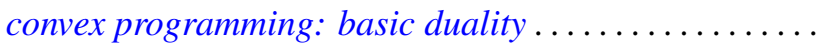

Alban J. Roques, Local evolution systems in general Banach spaces ..

I. Bert Russak, An indirect sufficiency proof for problems with bounded state variables.

Richard Alexander Sanerib, Jr., Ultrafilters and the basis property. .

H. A. Seid, The decomposition of multiplication operators on $L_{p}$-spaces . .

Franklin D. Tall, The density topology .................. 